\title{
LOCAL CONNECTIVITY AND MAPS ONTO NON-METRIZABLE ARCS
}

\author{
J. NIKIEL \\ American University of Beirut \\ Beirut, Lebanon \\ nikiel@layla.aub.ac.lb \\ and \\ L.B. TREYBIG \\ Texas A \& M University \\ College Station, Texas 77843-3368, USA \\ treybig@math.tamu.edu \\ and \\ H.M. TUNCALI \\ Nipissing University \\ North Bay, Ontario, Canada P1B 8L7 \\ muratt@einstein.unipissing.ca \\ (Received December 15, 1995 and in revised form July 6, 1996)
}

\begin{abstract}
Three classes of locally connected continua which admit sufficiently many maps onto non-metric arcs are investigated. It is proved that all continua in those classes are continuous images of arcs and, therefore, have other quite nice properties.
\end{abstract}

KEY WORDS AND PHRASES: arc, locally connected continuum, monotonically normal, rim-countable, rim-finite, rim-metrizable, rim-scattered

1991 AMS SUBJECT CLASSIFICATION CODES: Primary 54F15, Secondary 54C05 $54 \mathrm{~F} 05$

\section{INTRODUCTION}

Let $\mathcal{C}$ denote the class of all Hausdorff continuous images of ordered continua. In the last three decades the class $\mathcal{C}$ has been studied extensively by a number of authors (see e.g. [2], [4], [6-8], [11-13], [16-22], [26] and [27]). Two results from this study have suggested that the investigation could naturally be extended to the larger class $\mathcal{R}_{M}$ of all rim-metrizable, locally connected continua. Namely, (1) in [8] in 1967 Mardesić proved that each element of $\mathcal{C}$ has a basis of open $F_{\sigma}$-sets with metrizable boundaries, and (2) in [4] in 1991 Grispolakis, Nikiel, Simone and Tymchatyn showed that if a set $P$ is irreducible with respect to the property of being a compact set which separates the element $X$ of $\mathcal{C}$, then $P$ is metrizable. 
In his 1989 thesis [23] and two subsequent papers [24] and [25] Tuncali began an investigation of the class $\mathcal{R}_{M}$ and continuous images of elements of that class. He showed that Treybig's product theorem of [18] which holds in $\mathcal{C}$ is no longer valid in $\mathcal{R}_{M}$. However, he proved that Mardešić's theorem for $\mathcal{C}$ on preservation of weight by light mappings is true in $\mathcal{R}_{M},[25]$. He also considered the class $\mathcal{R}_{S}$ of all rim-scattered, locally connected continua, and the class $\mathcal{R}_{C}$ of all rim-countable, locally connected continua. Later, Nikiel, Tuncali and Tymchatyn gave an example to show that $\mathcal{R}_{C}$ is not a subclass of $\mathcal{C}$, [15]. Then, recently the authors of this paper showed the the continuous image of an element of $\mathcal{R}_{M}$ need not be in $\mathcal{R}_{M}$, [14]. Furthermore, Drozdovsky and Filippov proved in [3] that $\mathcal{R}_{S}$ is a larger class of spaces than $\mathcal{R}_{C}$.

Also, in 1973 Heath, Lutzer and Zenor, [5], showed that every linearly ordered ordered topological space and each of its Hausdorff continuous and closed images are monotonically normal. In [10] in 1986 Nikiel asked if every monotonically normal compactum is the continuous image of a compact ordered space. That problem still remains open. In what follows we let $\mathcal{R}_{M N}$ denote the class of monotonically normal, locally connected continua. Our first result is the following:

THEOREM 1. If $X \in \mathcal{R}_{M} \cup \mathcal{R}_{S} \cup \mathcal{R}_{M N}$ and for each pair of points $a, b \in X$ there exists a continuous onto map $f: X \rightarrow[c, d]$ such that $f(a)=c, f(b)=d$ and $[c, d]$ is a non-metrizable arc, then $X \in \mathcal{C}$.

We note that a large class of examples satisfying the properties of $X$ above can be constructed as follows: In [1] in 1945 Arens studied the class $\mathcal{L}$ of linear homogeneous continua, that is the class of arcs which are order isomorphic to each of their subarcs. Arens showed, that up to a homeomorphism, there exist at least $\aleph_{1}$ members of $\mathcal{L}$, including the real numbers interval $[0,1]$. Thus, some spaces $X$ as in Theorem 1 could be obtained by pasting together copies of any $Z \in \mathcal{L}$.

If a subset $B$ of a space $P$ contains no dense-in-itself, non-empty subset, we say that $B$ is scattered.

In this paper the definition of monotone normality we use is an equivalent one given in Lemma 2.2 (a) of [5]. It says that a space $P$ is monotonically normal provided there is an operator $G$ which assigns to each ordered pair $(S, T)$ of mutually separated subsets of $P$ an open set $G(S, T)$ such that

(i) $S \subset G(S, T) \subset \operatorname{cl}(G(S, T)) \subset P-T$, and

(ii) if $\left(S^{\prime}, T^{\prime}\right)$ is also a pair of mutually separated sets such that $S \subset S^{\prime}$ and $T^{\prime} \subset T$, then $G(S, T) \subset G\left(S^{\prime}, T^{\prime}\right)$.

PROOF OF THEOREM 1. Suppose that $X$ is not hereditarily locally connected. Then, there exists a subcontinuum $C$ of $X$ such that $C$ fails to be connected im kleinen at the point p. Utilizing the ideas in Theorem 11, p. 90, of Moore [9], there exists a connected open set $U$ containing $p$, a sequence $R_{1}, R_{2}, R_{3}, \ldots$ of connected open in $X$ sets containing $p$, and a sequence 
$G_{1}, G_{2}, G_{3}, \ldots$ of continua such that

(1) $U \supset \overline{R_{1}} \supset R_{1} \supset \overline{R_{2}} \supset R_{2} \supset \ldots$;

(2) $G_{n} \cap R_{n} \neq \emptyset$ and $G_{n} \cap R_{n+1}=\emptyset$ for $n=1,2,3, \ldots$;

(3) each $G_{n}$ is a component of $\bar{U} \cap C$ and $G_{n} \cap \mathrm{bd}(U) \neq \emptyset$ for $n=1,2,3, \ldots$; and

(4) $G_{n} \cap G_{m}=\emptyset$ if $n \neq m$, and there exist mutually exclusive open sets $V_{1}, V_{2}, V_{3}, \ldots$ such that $G_{n} \subset V_{n}$ for $n=1,2,3, \ldots$

For each positive integer $n$ let $H_{n}$ be a component of $G_{n}-R_{1}$ which intersects bd $\left(R_{1}\right)$ and $\mathrm{bd}(U)$, and let $s_{n} \in H_{n} \cap \mathrm{bd}\left(R_{1}\right)$ and $t_{n} \in H_{n} \cap \mathrm{bd}(U)$. Let $H_{0}$ denote the limiting set of the sequence $H_{1}, H_{2}, H_{3}, \ldots$; which by definition is the set of all $x$ such that every open set containing $x$ intersects infinitely many sets $H_{n}$.

Let $L_{1}$ (resp. $L_{2}$ ) denote the limiting set of $\left\{s_{1}\right\},\left\{s_{2}\right\},\left\{s_{3}\right\}, \ldots$ (resp. $\left\{t_{1}\right\},\left\{t_{2}\right\},\left\{t_{3}\right\}, \ldots$ ). There exists $(s, t) \in L_{1} \times L_{2}$ so that if $V$ is a neighborhood of $s$ and $W$ is a neighborhood of $t$, then $\left(s_{n}, t_{n}\right)$ belongs to $V \times W$ for infinitely many $n$.

We shall show that some component of $H_{0}$ contains $\{s, t\}$. If not, then $H_{0}$ is the union of two mutually separated sets $S$ and $T$ such that $s \in S$ and $t \in T$. There exist disjoint open sets $V$ and $W$ so that $S \subset V$ and $T \subset W$. Then $\left(s_{n}, t_{n}\right)$ belongs to $V \times W$ for infinitely many $n$. Since each $H_{n}$ is a continuum, $H_{n} \cap(X-(V \cup W)) \neq \emptyset$ for infinitely many $n$. It follows that some point of $H_{0}$ lies in $X-(V \cup W)$, a contradiction.

Let $f: X \rightarrow[c, d]$ be a continuous map onto a non-metrizable arc $[c, d]$, where $f(s)=c$ and $f(t)=d$. There is an increasing sequence $n_{1}, n_{2}, n_{3}, \ldots$ of positive integers such that

(1) $f\left(s_{n_{i}}\right) \geq f\left(s_{n_{1+1}}\right)$ and $f\left(t_{n_{i}}\right) \leq f\left(t_{n_{i+1}}\right)$ for $i=1,2, \ldots$;

(2) $f\left(s_{n_{1}}\right) \rightarrow c$ and $f\left(t_{n_{i}}\right) \rightarrow d$; and

(3) $\left[f\left(s_{n_{i}}\right), f\left(t_{n_{i}}\right)\right]$ is not metrizable for $i=1,2, \ldots$

Let $c^{\prime}=f\left(s_{n_{1}}\right)$ and $d^{\prime}=f\left(t_{n_{1}}\right)$.

Our proof now divides into three cases.

CASE 1. $X \in \mathcal{R}_{M}$. For each $n \geq 2$ let $M_{n}$ denote a metrizable closed set lying in $X-\bigcup_{k=1}^{n} H_{k}$ such that if $1 \leq i<j \leq n$, then $H_{i}$ and $H$, are separated in $X$ by $M_{n}$. Let $D_{n}$ denote a countable set dense in $M_{n}$ for $n=2,3, \ldots$ We intend to show that $f\left(\bigcup_{k=2}^{\infty} D_{k}\right)$ is dense in $[c, d]$, which would mean that $[c, d]$ is separable, and therefore metric, a contradiction.

Let $x \in] c, d[$ and let $c<u<x<v<d$ in the natural ordering of $[c, d]$. The components of $f^{-1}(] u, v[)$ which have limit points in both $f^{-1}(u)$ and $f^{-1}(v)$ can be labeled $P_{1}, P_{2}, \ldots, P_{n_{0}}$. Let $N_{0}$ be an integer such that if $i \geq N_{0}$ then $s_{n_{1}} \in f^{-1}\left(\left[c, u[)\right.\right.$ and $\left.\left.t_{n_{1}} \in f^{-1}(] v, d\right]\right)$. There exist two of $N_{0}, N_{0}+1, \ldots, N_{0}+n_{0}$, say $i$ and $j$, such that $H_{n}$, and $H_{n}$, both intersect the same $P_{l}$, which must then intersect some $D_{m}$. Therefore, $\bigcup_{k=2}^{\infty} f\left(D_{k}\right)$ intersects $] u, v[$.

CASE 2. $X \in \mathcal{R}_{M N}$. For each $i=1,2, \ldots$ let $Q_{i}$ denote a component of $H_{n_{i}} \cap f^{-1}\left(\left[c^{\prime}, d^{\prime}\right]\right)$ which intersects $f^{-1}\left(c^{\prime}\right)$ and $f^{-1}\left(d^{\prime}\right)$, and let $Q_{0}$ denote the limiting set of $Q_{1}, Q_{2}, Q_{3}, \ldots$ We note that some component of $Q_{0}$ intersects both $f^{-1}\left(c^{\prime}\right)$ and $f^{-1}\left(d^{\prime}\right)$ since every map onto an arc is weakly confluent. 
By Remark 2.3 (c) of [5], $Z=\bigcup_{n=0}^{\infty} Q_{n}$ is monotonically normal; so let $\mathcal{G}$ be a monotone normality operator on $Z$ as in the earlier definition. For each closed set $F$ in $\left[c^{\prime}, d^{\prime}\right]$ let $Q_{F}=$ $\left\{x: f(x) \in F\right.$ and $\left.x \in Z-Q_{0}\right\}$, and let $R_{F}=\left\{x: f(x) \in\left[c^{\prime}, d^{\prime}\right]-F\right.$ and $\left.x \in Q_{0}\right\}$. Now, $Q_{F}$ and $R_{F}$ are mutually separated subsets of $Z$; so for each positive integer $n$, let $T(F, n)=\left\{y \in\left[c^{\prime}, d^{\prime}\right]: y=f(x)\right.$ for some $\left.x \in Q_{n} \cap \mathcal{G}\left(Q_{F}, R_{F}\right)\right\}$. It can be shown that $T$ is a stratification for $\left[c^{\prime}, d^{\prime}\right]$. Since each stratifiable compact space is metrizable, $\left[c^{\prime}, d^{\prime}\right]$ is metrizable, a contradiction.

CASE 3. $X \in \mathcal{R}_{S}$. For each $i=1,2,3, \ldots$ let $K_{\imath}$ denote a component of $H_{n} \cap f^{-1}\left(\left[c^{\prime}, d^{\prime}\right]\right)$ which intersects $f^{-1}\left(c^{\prime}\right)$ and $f^{-1}\left(d^{\prime}\right)$.

We have to consider some subcases.

CASE 3A. $\left[c^{\prime}, d^{\prime}\right]$ contains uncountably many mutually exclusive open sets.

CASE $3 A_{1} \cdot\left[c^{\prime}, d^{\prime}\right]$ does not satisfy the first axiom of countability. Thus, without loss of generality, assume that there is a subset $\left\{d_{\alpha}: \alpha<\omega_{1}\right\}$ of $\left[c^{\prime}, d^{\prime}\right]$ such that $\alpha_{1}<\alpha_{2}$ implies that $d_{\alpha_{1}}<d_{\alpha_{2}}$ in $\left[c^{\prime}, d^{\prime}\right]$, and $d_{\alpha} \rightarrow d^{\prime}$.

Let $K_{0}$ denote the limiting set of $K_{1}, K_{2}, K_{3}, \ldots$ Let $Q$ denote a component of $K_{0}$ which intersects both $f^{-1}\left(c^{\prime}\right)$ and $f^{-1}\left(d^{\prime}\right)$. For each $\alpha<\omega_{1}$ let $W_{\alpha}$ denote a connected open set such that $W_{\alpha}$ contains a point $x_{\alpha}$ of $Q \cap f^{-1}(] d_{\alpha}, d_{\alpha+1}[)$, and $\overline{W_{\alpha}} \subset f^{-1}(] d_{\alpha}, d_{\alpha+1}[)$.

There exists a positive integer $n_{0}$ and a cofinal subsequence $\left\{d_{\alpha_{\beta}}\right\}$ of $d_{\alpha}$ such that $W_{\alpha_{\theta}} \cap$ $K_{n_{0}} \neq \emptyset$ for all $\alpha_{\beta}$. For each $\gamma<\omega_{1}$ let $L_{\gamma}$ denote the closure of the set $\bigcup_{\beta \geq \gamma} W_{\alpha_{\beta}}$. Let $L=\bigcap_{\gamma<\omega_{1}} L_{\gamma}$. Observe that if $y \in L$, then each open neighborhood of $y$ intersects uncountably many sets $W_{\alpha_{\rho}}$. Let $W$ be a component of $L$. Note that $W \cap K_{n_{0}} \neq \emptyset \neq Q \cap W$ and $W \subset f^{-1}\left(d^{\prime}\right)$. Thus, $W$ is a non-degenerate continuum.

Let $M_{0}$ and $M_{1}$ be connected open sets such that $\overline{M_{0}} \cap \overline{M_{1}}=\emptyset$ and $M_{2} \cap W \neq \emptyset$ for $i=0,1$. Let $\mathcal{G}_{1}=\left\{M_{0}, M_{1}\right\}$.

Now suppose that $\mathcal{G}_{n}$ has been chosen and consists of $2^{n}$ mutually exclusive connected open sets such that if $G, G^{\prime} \in \mathcal{G}_{n}$ and $G \neq G^{\prime}$, then $\bar{G} \cap \overline{G^{\prime}}=\emptyset$ and $G \cap W \neq \emptyset \neq G^{\prime} \cap W$. For each $G^{\prime} \in \mathcal{G}_{n}$ let $G_{0}^{\prime}$ and $G_{1}^{\prime}$ be mutually exclusive connected open sets such that $\overline{G_{0}^{\prime}} \cap \overline{G_{1}^{\prime}}=\emptyset$, $\overline{G_{0}^{\prime}} \cup \overline{G_{1}^{\prime}} \subset G^{\prime}$ and $G_{0}^{\prime} \cap W \neq \emptyset \neq G_{1}^{\prime} \cap W$. Let $\mathcal{G}_{n+1}=\left\{F: F=G_{0}^{\prime}\right.$ or $F=G_{1}^{\prime}$ for some $\left.G^{\prime} \in \mathcal{G}_{n}\right\}$. For each $n$ let $H_{n}^{\prime}=\bigcup \mathcal{G}_{n}$ and let $H=\bigcap_{n=1}^{\infty} H_{n}^{\prime}$.

There exists $\delta_{0}<\omega_{1}$ such that $G^{\prime} \cap f^{-1}\left(d_{\delta_{0}}\right) \neq \emptyset$ for each $G^{\prime} \in \bigcup_{j=1}^{\infty} \mathcal{G}_{j}$. There exists a closed scattered set $S$ in $X$ which separates $f^{-1}\left(\left[c, d_{\delta_{0}}\right]\right)$ from $f^{-1}\left(d^{\prime}\right)$. However, $S \cap H$ contains a perfect set because $S \cap H$ can be mapped onto a Cantor set, and it is well known that a scattered set cannot be mapped continuously onto a perfect set. This is a contradiction.

CASE 3A $\mathbf{A}_{2} .\left[c^{\prime}, d^{\prime}\right]$ satisfies the first axiom of countability at each point. Let \{]$c_{\alpha}, d_{\alpha}[$ : $\left.\alpha<\omega_{1}\right\}$ denote an uncountable collection of mutually exclusive open intervals in $] c^{\prime}, d^{\prime}[$. Using the local connectivity of $X$ we find that for each $\alpha$ there exists only a finite number, say $n_{\alpha}$, of components of $f^{-1}(] c_{\alpha}, d_{\alpha}[)$ which have limit points in both $f^{-1}\left(c_{\alpha}\right)$ and $f^{-1}\left(d_{\alpha}\right)$. Some integer $N_{0}=n_{\alpha}$ repeats for uncountably many $\alpha$ 's; so we may suppose without loss of generality that 
$n_{\alpha}=N_{0}$ for each $\alpha<\omega_{1}$.

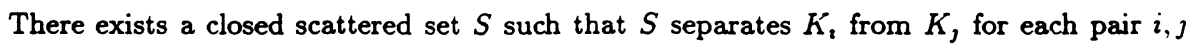
such that $1 \leq i<j \leq N_{0}+1$. Thus, since for each $\alpha$, each set $K_{\imath}$ where $1 \leq i \leq N_{0}+1$ has the property that some component of $K_{z} \cap f^{-1}\left(j c_{\alpha}, d_{\alpha} l\right)$ has limit points in both $f^{-1}\left(c_{\alpha}\right)$ and $f^{-1}\left(d_{\alpha}\right)$, it follows that $S$ must intersect each $f^{-1}(] c_{\alpha}, d_{\alpha}[)$.

Since $\left[c^{\prime}, d^{\prime}\right]$ is first countable, there exist collections $\mathcal{G}_{1}, \mathcal{G}_{2}, \mathcal{G}_{3}, \ldots$ such that (1) each $\mathcal{G}_{n}$ consists of $2^{n}$ mutually exclusive closed intervals in $\left[c^{\prime}, d^{\prime}\right]$, and (2) each element of each $\mathcal{G}_{n}$ contains exactly two elements of $\mathcal{G}_{n+1}$ and contains uncountably many elements of \{\}$c_{\alpha}, d_{\alpha}$ l: $\left.\alpha<\omega_{1}\right\}$.

For each positive integer $n$ let $L_{n}^{\prime}=\bigcup \mathcal{G}_{n}$, and let $L^{\prime}=\bigcap_{n=1}^{\infty} L_{n}^{\prime}$. We find that $S \cap f^{-1}\left(L^{\prime}\right)$ contains a perfect set, a contradiction.

CASE 3B. $\left[c^{\prime}, d^{\prime}\right]$ is not metrizable and does not contain uncountably many mutually exclusive open sets (i.e., it is a Souslin line). Thus, $\left[c^{\prime}, d^{\prime}\right]$ satisfies the first axiom of countability. If there exists a collection of metrizable open intervals whose union is dense in $\left[c^{\prime}, d^{\prime}\right]$, we find that $\left[c^{\prime}, d^{\prime}\right]$ is metrizable since it is separable. Hence, without loss of generality we may assume that $\left[c^{\prime}, d^{\prime}\right]$ contains no metrizable subinterval.

Similarly as above, for each $] x, y\left[\subset\left[c^{\prime}, d^{\prime}\right]\right.$ we let $n_{x y}$ denote the number of components of $f^{-1}(] x, y[)$ with limit points in both $f^{-1}(x)$ and $f^{-1}(y)$.

CASE $3 B_{1}$. Suppose there exists a positive integer $N_{0}$ and a subinterval $] x, y\left[\right.$ of $\left[c^{\prime}, d^{\prime}\right]$ such that if $x \leq z<w \leq y$, then $n_{z w} \leq N_{0}$. Let $S$ be a closed scattered set such that if $1 \leq i<j \leq N_{0}+1$, then $S$ separates $K_{2}$ from $K_{j}$. Using the ideas from Case $3 \mathrm{~A}_{2}$ we find that if $x \leq z<w \leq y$, then $S \cap f^{-1}(] z, w[) \neq \emptyset$. Therefore, $f(S) \supset[x, y]$, which contradicts the well-known fact that a scattered compactum can not be mapped onto a perfect set.

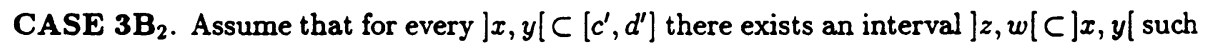
that $n_{z w}>n_{x y}$.

For each positive integer $n$ let $\mathcal{G}_{n}$ be maximal relative to the property of being a collection of mutually exclusive open intervals lying in $\left[c^{\prime}, d^{\prime}\right]$ such that if $] x, y\left[\in \mathcal{G}_{n}\right.$ then $n_{x y}=n$. Note that each $\mathcal{G}_{n}$ is at most countable. Let $S_{n}$ denote the set of all end-points of intervals which belong to $\mathcal{G}_{n}$. We are going to show that $\bigcup_{n=1}^{\infty} S_{n}$ is dense in $\left[c^{\prime}, d^{\prime}\right]$, and thus obtain a contradiction.

Let $] x, y\left[\subset\left[c^{\prime}, d^{\prime}\right]\right.$. There exists $] z, w[\subset] x, y\left[\right.$ such that $n_{z w}>n_{x y}$. Thus, $x \neq z$ or $y \neq w$. By maximality of $\mathcal{G}_{n_{z w}}$, there exists $] s, t\left[\in \mathcal{G}_{n_{x w}}\right.$ such that $] s, t[\cap] z, w[\neq \emptyset$. But $] s, t[\not \supset] x, y[$, and so $s \in] x, y[$ or $t \in] x, y\left[\right.$. Therefore, the set $\bigcup_{n=1}^{\infty} S_{n}$ is dense in $\left[c^{\prime}, d^{\prime}\right]$, a contradiction.

The consideration of subcases 1,2 and 3 is concluded and we return now to the main proof. Since $X$ is hereditarily locally connected, it is the continuous image of an arc by [12].

THEOREM 2. If $X$ is as in Theorem 1, then

(a) $X$ is rim-finite,

(b) every subcontinuum $G$ of $X$ has the property that some point or a pair of points separates $G$, and 
(c) each closed set irreducible with respect to the property of being a compact set which separates $X$ is metrizable.

PROOF. The claims (a), (b) and (c) follow from [19], [18] and [4], respectively, because $X$ contains no non-degenerate metric continuum.

Given a locally connected continuum $X$, for each pair of distinct points $a, b$ of $X$ let $[X, a, b]$ denote the class of all continuous maps $f: X \rightarrow P$ such that $P=f(X)$ is a non-metric arc with end-points $c$ and $d$ and $f(a)=c$ and $f(b)=d$. Also, introduce a relation $\sim$ on $X$ in the following way: $a \sim b$ if and only if $a=b$ or $[X, a, b]=\emptyset$.

THEOREM 3. Suppose that $X$ is a locally connected continuum. Then $\sim$ is an equivalence relation on $X$, and if $X$ also satisfies the first axiom of countability, then equivalence classes of $\sim$ are closed and the set $\mathcal{E}$ of equivalence classes of $\sim$ is upper semi-continuous.

PROOF. $\sim$ is easily seen to be reflexive and symmetric, so suppose that $a \sim b$ and $b \sim c$ hold, but that there exists $f \in[X, a, c]$ such that $f(X)$ is a non-metric arc $[d, e]$ with $f(a)=d$ and $f(c)=e$.

CASE 1. $f(b)=d$. Then $f \in[X, b, c]$, a contradiction.

CASE 2. $f(b)=e$ - analogous to Case 1 .

CASE 3. $d<f(b)<e$. Then one of the arcs $[d, f(b)]$ and $[f(b), e]$ is non-metric, so suppose $[d, f(b)]$ is non-metric. Define $r:[d, e] \rightarrow[d, f(b)]$ so that $r(x)=x$ if $x \in[d, f(b)]$ and $r(x)=f(b)$ if $x \in[f(b), e]$. Clearly, $r \circ f \in[X, a, b]$, a contradiction.

Let us now show that each equivalence class $G \in \mathcal{E}$ is closed if $X$ is first countable. Let $G \in \mathcal{E}$ and suppose that $x \in \bar{G}-G$. There exists a countable basis $U_{1}, U_{2}, \ldots$ of open neighborhoods of $x$ in $X$ and a sequence $x_{1}, x_{2}, \ldots$ of points of $G$ such that $x_{i} \in U_{i}$ for $i=1,2, \ldots$ Let $f: X \rightarrow[c, d]$ be a continuous map onto a non-metric arc $[c, d]$, where $f\left(x_{1}\right)=c$ and $f(x)=d$. Since each $\left[f\left(x_{1}\right), f\left(x_{i}\right)\right]$ is a metric subarc of $[c, d]$, it follows that $[c, d]$ is the closure of a countable union of metric arcs. Consequently, $[c, d]$ is separable, and therefore metrizable, a contradiction. Thus $G$ is closed in $X$.

It remains to show that $\mathcal{E}$ is upper semi-continuous if $X$ is first countable. Let the element $G$ of $\mathcal{E}$ be a subset of an open set $U$. Suppose that for each open set $V$ such that $G \subset V \subset U$, there is an element $G_{V}$ of $\mathcal{E}$ so that $V \cap G_{V} \neq \emptyset$ and $G_{V} \not \subset U$. Thus, for some point $x$ of $G$ there is a countable basis $U_{1}, U_{2}, \ldots$ of open neighborhoods of $x$ such that for each $U_{2}$ there is an element $G_{1}$ of $\mathcal{E}$ with the property that $G_{2} \cap U_{\imath} \neq \emptyset \neq G_{i} \cap(X-U)$.

There is a point $y$ of $X-U$ so that every neighborhood of $y$ intersects $G_{i}$ for infinitely many $i$. We may assume without loss of generality that there exists $y_{\mathrm{z}} \in G_{\mathrm{z}} \cap(X-U)$ for each $i$, and that the points $y_{i}$ converge to $y$. Let $z_{i} \in U_{i} \cap G_{i}$ for $i=1,2, \ldots$

There exists $f \in[X, x, y]$ such that $f: X \rightarrow[c, d]$, where $[c, d]$ is a non-metric arc, $f(x)=c$ and $f(y)=d$. Since the points $f\left(z_{i}\right)$ converge to $c$, and the points $f\left(y_{i}\right)$ converge to $d$, and each $\operatorname{arc}\left[f\left(z_{z}\right), f\left(y_{z}\right)\right]$ is metric, we find that $[c, d]$ is metric - a contradiction. 
THEOREM 4. Suppose that $X \in \mathcal{R}_{M} \cup \mathcal{R}_{S} \cup \mathcal{R}_{M N}$ and $X$ is first countable. Let $\mathcal{H}$ be the family of all components of sets in $\mathcal{E}$. Then $X / \mathcal{H}$ is the continuous image of an arc.

PROOF. Since $\mathcal{E}$ is upper semi-continuous, $\mathcal{H}$ is upper semi-continuous as well (see e.g. [28]). Thus, $\mathcal{H}$ is an upper semi-continuous decomposition of $X$ into closed sets and the quotient space $X / \mathcal{H}$ is a locally connected continuum.

If $X / \mathcal{H}$ is hereditarily locally connected, we apply the main result of [12] to obtain the desired conclusion.

Otherwise, in $X / \mathcal{H}$ there is a subcontinuum $C$ such that $C$ fails to be connected im kleinen at a point $P$. There is thus an open set $W$ in $X / \mathcal{H}$ such that $P \in W$ but the component of $W \cap C$ containing $P$ contains no relatively open subset of $C$ containing $P$. Let $Q$ denote the element of $\mathcal{E}$ containing $P$. There is a closed subset $S$ of $X$ such that $S \subset \cup W-Q$ and $S$ separates $P$ from bd(UW) in $X$. Let $\phi: X \rightarrow X / \mathcal{H}$ denote the natural map and let $B=\phi(S)$. Let $U$ denote the component of $X / \mathcal{H}-B$ which contains $P$. Using the facts that $\sim$ is upper semi-continuous and that $Q \cap S=\emptyset$, we let $R_{1}, R_{2}, \ldots ; G_{1}, G_{2}, \ldots ; V_{1}, V_{2} \ldots$ be subsets of $X / \mathcal{H}$ similarly as in the proof of Theorem 1, except for the additional condition that no element of $\mathcal{E}$ intersects $\operatorname{cl}\left(\bigcup R_{1}\right)$ and $\mathrm{bd}(\bigcup U)$.

Now, let $s_{1}, s_{2}, \ldots$ and $t_{1}, t_{2}, \ldots$ be such that $s_{2} \in\left(\bigcup G_{\imath}\right) \cap\left(\bigcup b d\left(R_{1}\right)\right)$ and $t_{2} \in\left(\bigcup G_{\imath}\right) \cap$ $(\bigcup \operatorname{bd}(U))$ for $i=1,2, \ldots$ Since $X$ is first countable, we may assume without loss of generality that the points $s_{3}$ converge to some point $s$, and the points $t_{\mathrm{z}}$ converge to some point $t$, and the limiting set $L$ of $\bigcup G_{1}, \cup G_{2}, \cup G_{3}, \ldots$ is a continuum containing $s$ and $t$.

There is an $f \in[X, s, t]$ such that $f(X)$ is a non-metric $\operatorname{arc}[c, d]$ with $f(s)=c$ and $f(t)=d$. We may now obtain a contradiction as in the proof of Theorem 1.

ACKNOWLEDGMENT. H.M. Tuncali was partially supported by an NSREC grant.

\section{REFERENCES}

[1] Arens, R., "On the construction of linear homogeneous continua," Böl. Soc. Mat. Mexicana 2 (1945), 33-36.

[2] Cornette, J.L., "Image of a Hausdorff arc is cyclically extensible and reducible," Trans. Amer. Math. Soc. 199 (1974), 255-267.

[3] Drozdovsky S.A. and Filippov, V.V., "An example of a rim-scattered locally connected continuum which is not rim-countable (in Russian)," Mat. Sbornik 185, No. 10 (1994), 27-38.

[4] Grispolakis, J., Nikiel, J., Simone J.N. and Tymchatyn, E.D., "Separators in continuous images of ordered continua and hereditarily locally connected continua," Canadian Math. Bull. 36 (1993), 154-163.

[5] Heath, R.W., Lutzer D.J. and Zenor, P.L., "Monotonically normal spaces," Trans. Amer. Math. Soc. 178 (1973), 481-493.

[6] Mardesić, S., "On the Hahn-Mazurkiewicz theorem in non-metric spaces," Proc. Amer. Math. Soc. 11 (1960), 929-937.

[7] Mardešić, S., "Mapping ordered continua onto product spaces," Glasnik Mat. 15 (1960), 85-89. 
[8] Mardešić, S., "Images of ordered compacta are locally peripherally metric," Pacific J. Math. 23 (1967), 557-568.

[9] Moore, R.L., "Foundations of point set topology" (rev. ed.), Amer. Math. Soc. Colloquium Publications, vol. 13, Providence, RI, 1962.

[10] Nikiel, J., "Some problems on continuous images of compact ordered spaces," Questions Answers Gen. Topology 4 (1986/87), 117-128.

[11] Nikiel, J., "Images of arcs - a nonseparable version of the Hahn-Mazurkiewicz theorem," Fund. Math. 129 (1988), 91-120.

[12] Nikiel, J., "The Hahn-Mazurkiewicz theorem for hereditarily locally connected continua," Topology Appl. 32 (1989), 307-323.

[13] Nikiel, J., "On continuous images of arcs and compact orderable spaces," Topology Proc. 14 (1989), 163-193 and 279-280.

[14] Nikiel, J., Treybig, L.B. and Tuncali, H.M., "A rim-metrizable continuum," Proc. Amer. Math. Soc. 123 (1995), 281-286.

[15] Nikiel, J., Tuncali, H.M. and Tymchatyn, E.D., "A locally connected rim-countable continuum which is the continuous image of no arc," Topology Appl. 42 (1991), 83-93.

[16] Pearson, B.J., "Mapping an arc onto a dendritic continuum," Collog. Math. 30 (1974), 237-243.

[17] Simone, J.N., "Metric components of continuous images of ordered compacta," Pacific J. Math. 69 (1977), 269-274.

[18] Treybig, L.B., "Concerning continuous images of compact ordered spaces," Proc. Amer. Math. Soc. 15 (1964), 866-871.

[19] Treybig, L.B., "Concerning continua which are continuous images of compact ordered spaces," Duke Math. J. 32 (1965), 417-422.

[20] Treybig, L.B., "Separation by finite sets in connected, continuous images of ordered compacta," Proc. Amer. Math. Soc. 74 (1979), 326-328.

[21] Treybig, L.B., "A characterization of spaces that are the continuous image of an arc," Topology Appl. 24 (1986), 229-239.

[22] Treybig, L.B. and Ward, Jr., L.E., "The Hahn-Mazurkiewicz problem," in: Topology and order structures I, Math. Centre Tracts, vol. 142, Amsterdam, 1981, p. 95-105.

[23] Tuncali, H.M., "Some generalizations of the Hahn-Mazurkiewicz theorem," Ph.D. Thesis, University of Saskatchewan, Saskatoon, 1989.

[24] Tuncali, H.M., "Analogues of Treybig's product theorem," Proc. Amer. Math. Soc. 108 (1990), 855-858.

[25] Tuncali, H.M., "Concerning continuous images of rim-metrizable continua," Proc. Amer. Math. Soc. 113 (1991), 461-470.

[26] Ward, Jr., L.E., "A generalization of the Hahn-Mazurkiewicz theorem," Proc. Amer. Math. Soc. 58 (1976), 369-374.

[27] Ward, A.J., "Some properties of images of ordered compacta with special reference to topological limits," unpublished.

[28] Whyburn, G.T., "Analytic topology," Amer. Math. Soc. Colloquium Publications, vol. 28, Providence, RI, 1942. 


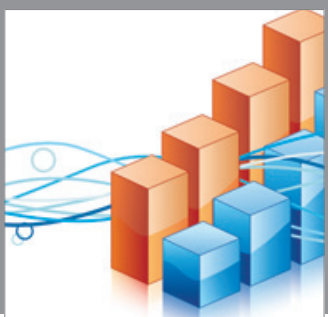

Advances in

Operations Research

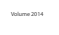

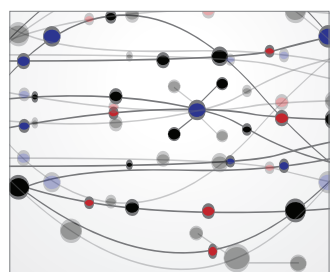

\section{The Scientific} World Journal
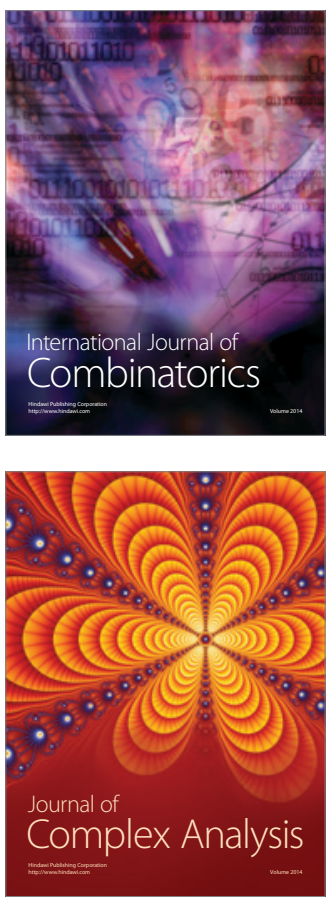

International Journal of

Mathematics and

Mathematical

Sciences
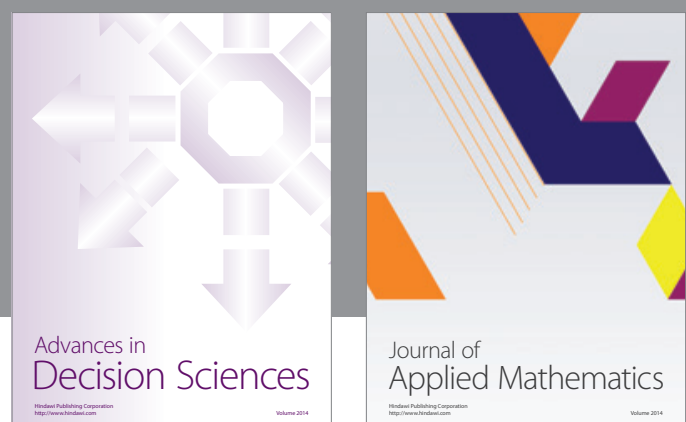

Journal of

Applied Mathematics
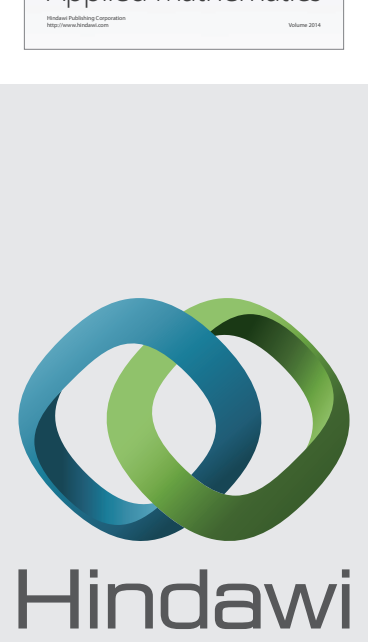

Submit your manuscripts at http://www.hindawi.com
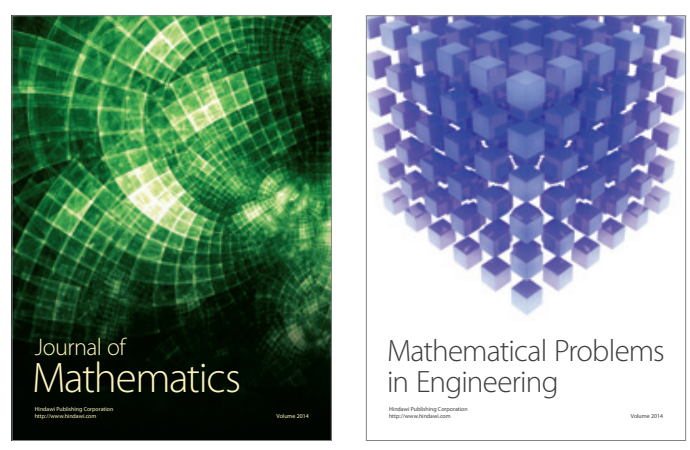

Mathematical Problems in Engineering
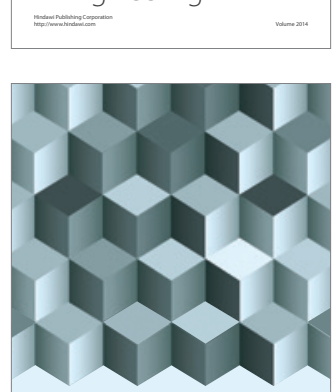

Journal of

Function Spaces
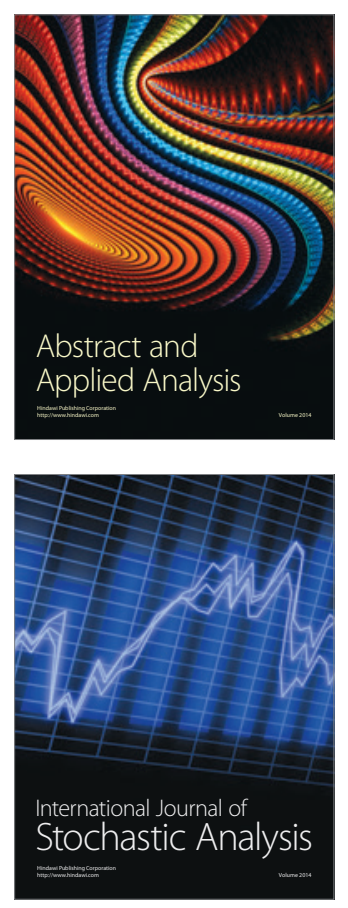

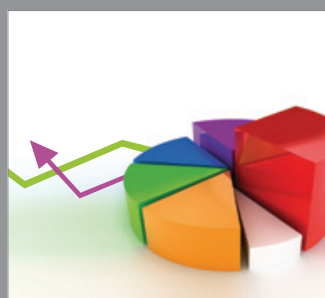

ournal of

Probability and Statistics

Promensencen
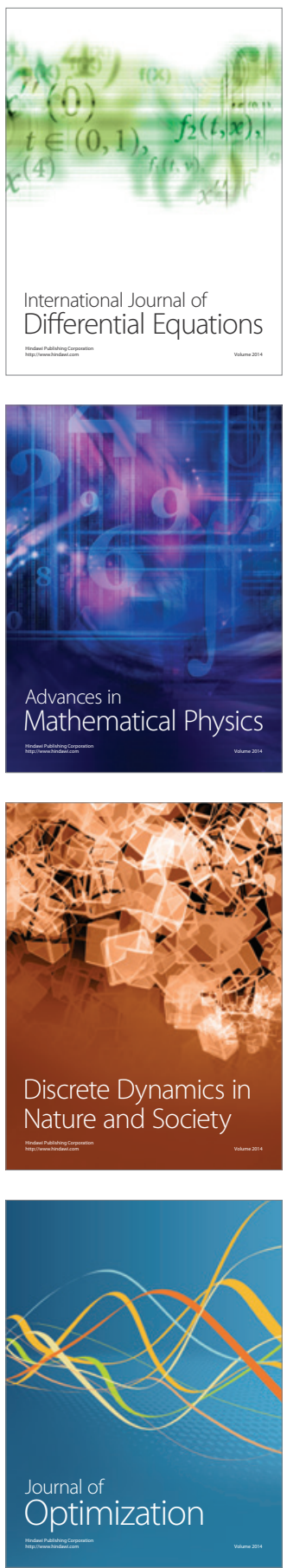